\title{
Desarrollo del Razonamiento Algebraico Elemental mediante Tareas de Recuento con Patrones
}

\author{
Developing Elementary Algebraic Reasoning through Counting with \\ Patterns Activities
}

\author{
Rosa Gaita* \\ http://orcid.org/0000-0002-7827-9262 \\ Miguel R. Wilhelmi** \\ https://orcid.org/0000-0002-6714-7184
}

\begin{abstract}
Resumen
Las tareas de recuento con patrones son un contexto privilegiado para el desarrollo del razonamiento algebraico. La complejidad ontosemiótica de las tareas permite identificar procesos de estudio para hacer progresar el desempeño en sucesivos niveles de algebrización, desde la manipulación meramente aritmética hasta una práctica con un nivel algebraico consolidado. El objetivo es doble: por un lado, elaborar indicadores que permitan valorar el desempeño de una práctica operatoria y discursiva en la resolución de tareas de recuento de patrones; por otro lado, proponer una secuencia que tenga en cuenta las restricciones epistemológicas, cognitivas y de enseñanza propias de estas prácticas. Un diseño pre-test y post-test mediante un cuestionario de preguntas abiertas y el análisis cualitativo de las respuestas por estudio de casos aporta información relevante sobre el desarrollo del razonamiento algebraico de los sujetos en los sucesivos niveles de algebrización. Se concluye que el Pensamiento Matemático Flexible es un indicador clave de la competencia algebraica de los sujetos, y se aporta una secuencia de enseñanza que parte de una estrategia inicial, basada en manipulaciones concretas y generalizaciones cercanas hasta el análisis de distintas expresiones analíticas.
\end{abstract}

Palabras-clave: Razonamiento Algebraico. Patrones. Enfoque Ontosemiótico. Pensamiento Matemático Flexible. Normas.

\begin{abstract}
The counting tasks with patterns are a privileged context for the development of algebraic reasoning. The ontosemiotic complexity of tasks allows the identification of study processes to improve performance in successive algebrization levels, from purely arithmetic manipulation to a practice with a consolidated algebraic level. The objective is twofold: on the one hand, to elaborate indicators that allow evaluating the performance of an operative and discursive practice in the task resolution of counting patterns. On the other hand, to propose a sequence that takes into account the epistemological, cognitive, and teaching restrictions common to these practices. A pre-test and post-test design is proposed using an open-ended questionnaire. A qualitative analysis is carried out through a case study, which provides relevant information on the development of the subjects' algebraic reasoning in the successive algebrization levels. It is concluded that Flexible Mathematical Thinking is a key indicator of the subjects' algebraic competence; we also provided a teaching sequence that start from a basic strategy based on concrete manipulations and close generalizations to the analysis of different analytical expressions.
\end{abstract}

\footnotetext{
* Doctora en Didáctica de la Matemática por la Universidad de Valladolid (UVA). Docente principal del Departamento de Ciencias de la Pontificia Universidad Católica del Perú (PUCP), Lima, Perú. Dirección postal: Avenida Universiraria 1801, San Miguel, Lima 32, Lima, Perú, C.P: 15088. E-mail: cgaita@pucp.edu.pe.

** Doctor en Matemáticas por la Universidad Pública de Navarra (UPNA). Profesor permanente del Departamento de Matemáticas de la UPNA, España. Dirección postal: Campus Arrosadia s/n, Pamplona, Navarra, España, C.P: 31006. E-mail: miguelr.wilhelmi@unavarra.es.
} 
Keywords: Algebraic Reasoning. Patterns. Onto-semiotic Approach. Flexible Mathematical Thinking. Norms.

\section{Adecuación de la actividad matemática a tareas de recuento con patrones}

La Didáctica del álgebra es un área prioritaria de investigación. Los congresos internacionales principales $\left(\mathrm{CERME}^{1}, \mathrm{RELME}^{2}, \mathrm{PME}^{3}\right)$ establecen grupos, talleres o monográficos de investigación sobre este ámbito. Además, el álgebra es un tópico utilizado de forma extensa para ejemplificar o articular discursos vinculados a aspectos epistemológicos (argumentación y prueba, visualización, representación, modelos etc.) o de intervención pedagógica o didáctica (evaluación, enseñanza y aprendizaje con tecnología, desarrollo docente etc.).

En este contexto, la comunidad Early Algebra se ha asentado en los últimos treinta años (KIERAN et al., 2016), aportando fundamentación teórica y datos experimentales sobre la conveniencia de superar la oposición aritmético-algebraico y sobre la necesidad de estructurar el currículo como un continuo epistemológico, antes que un paso de una actividad meramente aritmética (en Educación Primaria) a otra donde el álgebra se presenta como un producto acabado (en Educación Secundaria).

Así, se sostiene que "las relaciones matemáticas, los patrones y las estructuras aritméticas están en el centro de la actividad algebraica temprana" (KIERAN et al., 2016, p. 1). Es decir, no es preciso algebrizar ciertos contenidos matemáticos para ser susceptibles de ser enseñados.

Gascón, Bosch y Ruiz-Munzón (2017) realizan una revisión del desarrollo del álgebra elemental desde la Teoría Antropológica de lo Didáctico (TAD) en los últimos 25 años $^{4}$. Este desarrollo ha permitido establecer un modelo epistemológico de referencia (MER), cuyo fin último es la modelización algebraica de sistemas de todo tipo.

Godino y Burgos (2017) realizan, asimismo, una revisión del desarrollo del razonamiento algebraico elemental (RAE) desde el Enfoque Ontosemiótico del conocimiento y de la instrucción matemáticos $(\mathrm{EOS})^{5}$. El modelo del EOS se centra en la actividad del sujeto,

\footnotetext{
${ }^{1}$ Congress of the European Society for Research in Mathematics Education.

${ }^{2}$ Reunión Latinoamericana de Matemática Educativa.

${ }^{3}$ International Group for the Psychology of Mathematics Education.

${ }^{4}$ El trabajo aporta, además de un síntesis profunda y clara, una extensa bibliografía para profundizar en el álgebra elemental según la TAD, que puede completarse con el acceso a la página Web del grupo de investigación (http://www.atd-tad.org/).

${ }^{5}$ El trabajo aporta, además de un síntesis profunda y clara del RAE, referencias al desarrollo teórico en el EOS, que puede completarse con el acceso a su página Web (http://enfoqueontosemiotico.ugr.es/).
} 
de tal forma que una misma situación puede ser abordada según diferentes niveles de algebrización.

Así, el EOS (GODINO et al., 2012; GODINO et al., 2014a) prevé una evolución en distintos niveles de algebrización, teniendo en cuenta: a) la función y uso de los lenguajes involucrados, b) los procesos de particularización y generalización abordados y, por último, c) la dualidad particular/general (o ejemplar/tipo;) que determina el significado contextual de los objetos movilizados.

Así, los objetos varían desde la operación con números particulares, en el nivel exclusivamente aritmético (nivel 0), a la manipulación de incógnitas, ecuaciones, variables y funciones particulares, en un nivel consolidado de algebrización (nivel 3), produciéndose una introducción paulatina de extensivos transformados en diferentes grados y contextos. Esta estructura en niveles permite sobrepasar la dicotomía aritmética-álgebra.

Asimismo, es preciso tener en cuenta que tanto las etapas de algebrización propuestas por la TAD como los niveles de algebrización en el EOS suponen una ampliación progresiva de las herramientas y del campo de problemas abordables. En ambos casos, las etapas o niveles permiten un aumento progresivo del poder heurístico en la resolución de problemas.

El uso del modelo RAE se ha centrado en la promoción del razonamiento algebraico en profesorado en formación inicial, sin embargo, "no se han abordado aún las implicaciones del modelo de niveles RAE sobre la planificación curricular ni tampoco para distinguir posibles niveles de desarrollo cognitivo de los estudiantes" (GODINO; BURGOS, 2017, p. 50). El objetivo de este trabajo es progresar en estos aspectos, centrando el interés en el uso de patrones para el desarrollo del RAE y la determinación de propuestas de intervención.

Para ejemplificar el modelo se propone, a continuación, una tarea de recuento con patrones (GODINO et al., 2015a; GODINO et al., 2015b) (Figura 1).

Considera la siguiente sucesión de figuras.

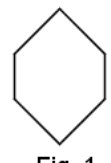

Fig. 1

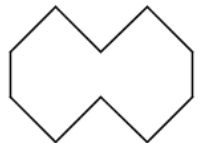

Fig. 2

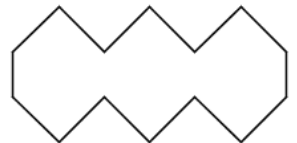

Fig. 3

Si se mantiene el mismo patrón:

1) Determina cuántos segmentos se requerirán para formar la figura 5.

2) Determina cuántos segmentos se requerirán para formar la figura 87.

Figura 1 - Tarea de recuentos con patrones

Fuente: Elaboración propia de esta investigación

La resolución del primer apartado se puede realizar por construcción efectiva del dibujo que ocupa el lugar 5. Si bien el apartado $2^{\circ}$, figura de la posición 87 , también se puede abordar 
mediante la construcción efectiva, este se propone con el objetivo de que los estudiantes busquen un método general, más eficaz y económico.

A continuación, se describen las soluciones de estudiantes A, B y C. El estudiante A (Figura 2) cuenta los segmentos de cada figura: en el primer caso se requieren $4+2$ segmentos, 4 de la celda (puntas) y 2 de los dos segmentos extremos verticales; en el segundo, $10(=4 \times 2$ +2 ; dos celdas de 4 y los extremos verticales); en el tercero, $14(=4 \times 3+2)$; y, finalmente, en el quinto, $22(=4 \times 5+2)$. Es decir, determina una regla general que distingue las celdas (que aportan 4 segmentos) de los extremos verticales (2). Así, la respuesta al apartado 2) es: $350=$ $4 \times 87+2$ segmentos; sin necesidad de recuento expreso.

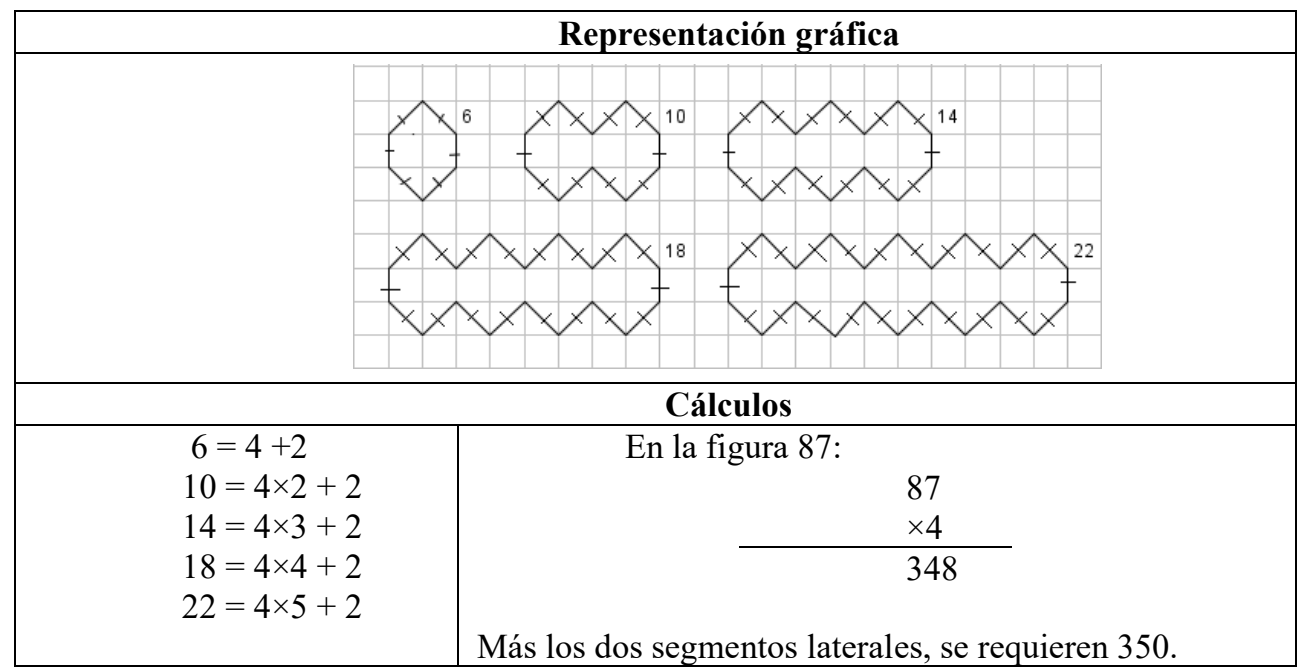

Figura 2 - Resolución gráfica del estudiante A

Fuente: Elaboración propia de esta investigación

El estudiante B señala que en la figura de la posición 1 se emplean 6 segmentos y que por cada figura adicional este número se incrementa en 6. Este estudiante no contrasta su solución con un recuento de algunos casos particulares y, por lo tanto, no observa la necesidad de suprimir los segmentos interiores (que se obtendrían al unir celdas). Concluye, erróneamente, que para la figura 5 se necesitan $30(=6 \times 5)$ segmentos y, para la figura 87,522 $(=6 \times 87)$ segmentos.

Por último, el estudiante $\mathrm{C}$ dibuja la figura 5 de la serie y cuenta los segmentos, sin identificar ninguna regularidad. Sin embargo, en la resolución del apartado 2, este estudiante hace dos agrupamientos: uno de 7 celdas, donde cuenta 30 segmentos, y otro de 10 celdas, con 42 segmentos. Entonces, concluye que la figura 87 se forma con 8 figuras de 10 celdas y una de 7, en total $366(=8 \times 42+30)$ segmentos. Luego hay que quitar los segmentos de las uniones que son $16(=8 \times 2)$. Es decir, son $350(=366-16)$ segmentos (Figura 3). 


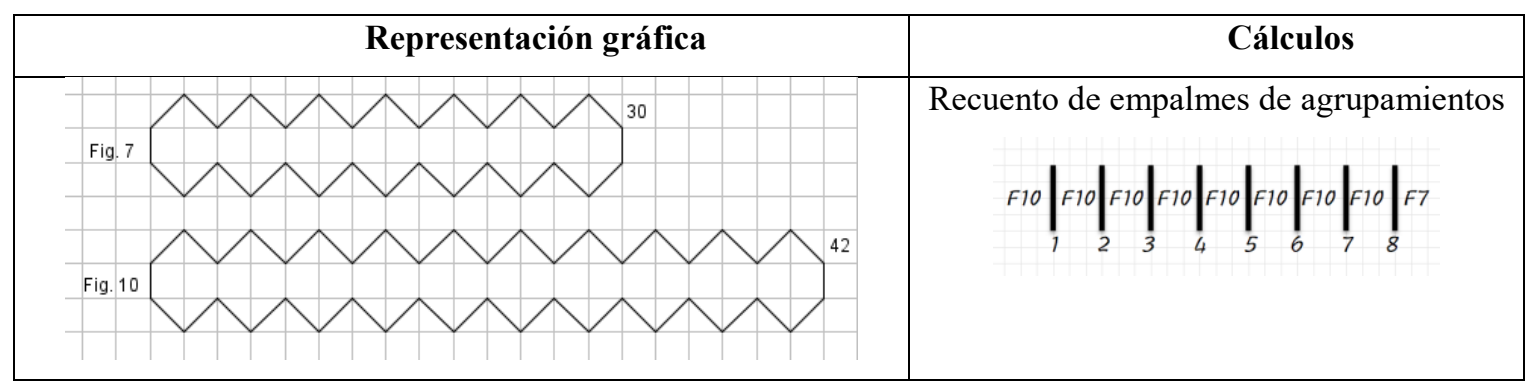

Figura 3 - Recuento de celdas por agrupamientos

Fuente: Elaboración propia de esta investigación

Los estudiantes A y C realizan adecuadamente la tarea, aportando para los dos apartados la solución correcta; pero, las respuestas denotan distintos niveles de generalización. Por otro lado, el estudiante B da una regla incorrecta, que es indicativa tanto de la importancia atribuida por este a la obtención de un criterio general de formación como de la falta de medios de control sobre la validez de la fórmula determinada. Más allá de si, las respuestas son correctas o no, sus soluciones presentan distintos rasgos de RAE.

Así, es necesario proponer procesos de estudio que permitan el progreso gradual entre distintos niveles de algebrización, desde una actividad puramente aritmética a otra en la que el álgebra esté consolidada. Para ello, las tareas sobre patrones son un contexto adecuado.

\footnotetext{
Los patrones o regularidades existen y aparecen de manera natural en las matemáticas. Pueden ser reconocidos, ampliados o generalizados. El mismo patrón se puede encontrar en muchas formas diferentes. Los patrones se encuentran en situaciones físicas, geométricas y numéricas (GODINO et al., 2014a, p. 202).
}

En la misma línea, Moss y London (2011) señalan que, con una instrucción apropiada, el estudio de patrones puede fomentar el razonamiento algebraico en estudiantes con diferentes niveles de habilidades matemáticas. Callejo y Zapatera (2014) indican que los problemas sobre patrones en los que el término general de la sucesión se puede asociar a una función lineal, cuadrática, etc., generan un escenario adecuado para hacer la transición de la aritmética al álgebra, introduciendo la noción de generalización a través de la búsqueda de patrones.

En este trabajo se discute cómo progresar en los distintos niveles de algebrización (GODINO et al., 2012, 2015a) en tareas de recuento con patrones. Para alcanzar este objetivo, en la sección 2 se describen los niveles de algebrización y se adaptan a tareas con patrones y se discute la adecuación de los métodos de resolución de tareas con patrones para la adquisición de conocimientos algebraicos y de un pensamiento matemático flexible (WILHELMI; GODINO; LACASTA, 2007a). En la sección 3 se describe la experimentación, que se desarrolla en la sección 4. En la sección 5, se propone una propuesta de intervención para el desarrollo del razonamiento algebraico mediante patrones con base en el estudio teórico y los datos experimentales. Por último, se realiza una síntesis, se aportan conclusiones y unas 
cuestiones abiertas.

\section{Adaptación de niveles de algebrización a tareas de construcción y análisis de patrones}

El razonamiento algebraico se desarrolla paulatinamente. Distintos autores han centrado sus investigaciones en el tránsito de la aritmética al álgebra, que supone una ruptura epistemológica y un reto cognitivo (KAPUT; CARRAHER; BLANTON, 2008). Blanton y Kaput (2011) proponen un recorrido para el desarrollo del álgebra en los primeros grados escolares (hasta los 9 años) a través del pensamiento funcional (functional thinking): desde la escuela elemental (3-7 años), donde los niños utilizan la representación tabular de funciones, establecen las primeras regularidades con números concretos; hasta que en $3^{\circ}$ primaria (8-9 años), se progresa en la simbolización y se establecen relaciones de covaración.

The elementary grades often incorporate meaningful imagery and concrete experiences to support conceptual development, they [...] can provide a rich, inquirybased atmosphere for introducing symbolic notation [...] The data illustrate [some] covariational and correspondence relationships, [that] these represents the critical kinds of experiences that children need in the earlier elementary grades in order to leverage deeper, more complex functional thinking in later elementary grades and beyond (BLANTON Y KAPUT, 2011, p. 14-15).

El modelo del RAE permite describir la práctica operativa y discursiva desarrollada por un sujeto en función de los objetos matemáticos involucrados. Por ello, se establece que un sujeto utiliza de manera eficaz las herramientas propias de un determinado nivel de RAE si es capaz de adaptarlas a la tarea, usando estas o las de niveles inferiores de manera flexible.

[El Pensamiento Matemático Flexible (PMF) permite el] tránsito rutinario entre diferentes modelos asociados a un objeto matemático, reconociendo las limitaciones propias de cada uno de ellos, [y estableciendo] nexos firmes entre dichos modelos y uno o varios contextos matemáticos, que determinan un control eficaz de la actividad y capacitan al sujeto para responsabilizarse matemáticamente de los resultados que produce (WILHELMI; GODINO; LACASTA, 2007a, p. 106).

Así, el PMF permite evaluar la resolución de las tareas según los niveles de algebrización en términos de eficacia y coste (WILHELMI; GODINO; LACASTA, 2007b). Por ejemplo, en tareas de patrones con un número concreto y reducido de objetos, la estrategia más eficaz es la determinación efectiva (respuesta por extensión); cuando el número de casos es elevado, por inducción empírica, se determina una regla general.

Por ello, la descripción de los niveles en la resolución de tareas de patrones debe tener en cuenta este uso flexible. En el Cuadro 1 se presentan los niveles RAE (GODINO et al., 2014a), adaptados a tareas sobre patrones. 


\begin{tabular}{|c|c|}
\hline Nivel & Descripción del nivel en tareas de construcción y análisis de patrones \\
\hline $\mathbf{0}$ & $\begin{array}{l}\text { La determinación de casos particulares se realiza a partir de representaciones concretas y la descripción } \\
\text { extensiva de las mismas. Los recuentos son explícitos, basados en cálculos numéricos o figuras. El análisis } \\
\text { y discusión se realiza en lenguaje natural, numérico, icónico o gestual. Eventualmente, intervienen } \\
\text { símbolos, que se refieren a objetos extensivos y suponen, en general, una forma abreviada de comunicar } \\
\text { información, pero en ningún caso involucran la manipulación simbólica, abstracta o general. }\end{array}$ \\
\hline 1 & $\begin{array}{l}\text { La determinación de casos particulares se realiza a partir de representaciones concretas y la descripción } \\
\text { extensiva de las mismas. A partir de recuentos explícitos, basados en cálculos numéricos o figuras, es } \\
\text { posible determinar el valor de patrones específicos en función del lugar que ocupan en la serie, sin recurrir, } \\
\text { nuevamente, al recuento efectivo ni a la representación del patrón completo (generalización cercana). El } \\
\text { análisis y discusión se realizan en lenguaje natural, numérico, icónico o gestual, que incluye expresiones } \\
\text { que se refieren a objetos generales y representan el caso general con un lenguaje no simbólico ni formal, } \\
\text { pero necesariamente explícito. Eventualmente, se establecen relaciones entre figuras en la serie o } \\
\text { propiedades del patrón. }\end{array}$ \\
\hline 2 & $\begin{array}{l}\text { La determinación de casos particulares se realiza a partir de representaciones concretas y descripción } \\
\text { extensiva de las mismas. A partir de recuentos, basados en cálculos numéricos y representaciones gráficas, } \\
\text { se determina el valor de patrones específicos en función del lugar que ocupan en la serie, representando el } \\
\text { patrón completo. El análisis y discusión se realizan, en primer lugar, en lenguaje natural, numérico, icónico } \\
\text { o gestual, y, en segundo lugar, se formaliza mediante símbolos, variables o parámetros, que refieren } \\
\text { objetos generales y representan el caso general, que puede ser utilizado para la descripción del método de } \\
\text { construcción del patrón. Se establecen relaciones entre figuras en la serie o propiedades del patrón, pero } \\
\text { no se manipula para ello la escritura formal, si no que se interpreta ésta mediante lenguaje natural o } \\
\text { numérico. }\end{array}$ \\
\hline 3 & $\begin{array}{l}\text { Se determina de la regla general y se expresa formalmente. Los casos particulares, a partir de } \\
\text { representaciones concretas y descripción extensiva de las mismas, pueden aparecer en un análisis previo } \\
\text { o como ejemplificación de la regla general. En casos simples, cuando la construcción efectiva es posible, } \\
\text { eficaz y poco costosa, se puede optar por ella, pero, en general, el análisis y discusión se realizan, en } \\
\text { primer lugar, en lenguaje simbólico literal formalizado, mediante símbolos, variables o parámetros, que } \\
\text { refieren objetos generales y representan el caso general. Ocasionalmente, en segundo lugar, se utiliza un } \\
\text { lenguaje natural, numérico, icónico o gestual en la descripción del método de construcción del patrón; este } \\
\text { uso tiene una función comunicativa (reforzar una aseveración general, explícitamente formulada) o } \\
\text { pedagógica (mostrar al docente el conocimiento extenso de la situación propuesta). Se establecen } \\
\text { relaciones entre figuras en la serie o propiedades del patrón. Además, si es necesario, se manipula la } \\
\text { escritura formal para obtener expresiones más simples, sin necesariamente analizar su relación con el } \\
\text { método de construcción, centrándose el trabajo en la manipulación simbólico-literal. }\end{array}$ \\
\hline
\end{tabular}

Cuadro 1 - Niveles de algebrización en tareas de construcción y análisis de patrones

Fuente: Elaboración propia de esta investigación

La resolución clásica de tareas con patrones se puede sistematizar de la siguiente forma: 1) análisis de casos particulares, 2) conjetura de una ley general de formación y 3) demostración de la ley general por un procedimiento formalizado. El primer paso suele conllevar la realización de un número reducido de casos, normalmente de los primeros elementos de la secuencia de patrones. Para el segundo, se determina una conjetura mediante inducción empírica, es decir, basándose en una regla percibida en los casos efectivamente realizados en el paso anterior. Por último, se demuestra la regla general por inducción matemática.

Este esquema clásico, aunque válido, desatiende la génesis del conocimiento de quienes se introducen en la realización de estas tareas, que no poseen ni la destreza suficiente en la manipulación simbólica ni los conocimientos teóricos necesarios. Necesitan evolucionar su práctica operatoria y discursiva; evolución que debe partir de procedimientos $\mathrm{y}$ argumentaciones previamente movilizados y de la determinación de su campo de validez, 
eficacia y coste de ejecución. De hecho, se distingue a los estudiantes competentes por su capacidad de análisis y selección de los procedimientos mejor adaptados a las tareas que se debe resolver, es decir, aquellos que poseen un pensamiento matemático flexible (PMF).

En este mismo sentido, Callejo y Zapatera (2014, p. 65) caracterizan la flexibilidad como la capacidad para "la modificación de las estrategias empleadas en la resolución de un problema cuando se modifica la demanda de la tarea, en estudiantes de educación secundaria obligatoria (12-16 años)”. De esta forma, los sujetos más capaces ajustan los procedimientos de recuento de situaciones con patrones a las distintas tareas que deben afrontar. Así, por ejemplo, procederán de manera distinta atendiendo a:

- $\quad$ El término de la serie solicitado. No es lo mismo la determinación de términos para los que la construcción efectiva es fácil, que otros para los que esta construcción precisa de una cantidad considerable de recursos cognitivos o temporales.

- Los recursos disponibles para la determinación de la regla general de formación y su demostración. No es lo mismo el conocimiento previo de un número finito de casos y el dominio de la representación mediante lenguaje simbólico-literal de fórmulas, que una situación abierta afrontada con lenguaje natural.

De esta forma, la valoración de respuestas de los estudiantes debe partir de la premisa siguiente: la sola respuesta algebraica no es indicadora, en sí misma, de un gran dominio de la situación, y el recurso a métodos aritméticos no es necesariamente una limitación. Con otras palabras, son los estudiantes que muestran un PMF, combinando métodos aritméticos y algebraicos, los que en general tienen el desempeño más alto.

Así, el estudiante A de la sección 1 muestra un PMF dado que tras un análisis concreto (aritmético y figural) establece una relación general. El estudiante B procede de forma similar, pero no introduce elementos de control de sus resultados y, por lo tanto, produce una solución rígida que no se apoya en un uso diferenciado de diferentes herramientas aritméticas o algebraicas. El estudiante $\mathrm{C}$ realiza un recuento sistemático de situaciones concretas, que articula para la construcción de figuras con un mayor número de celdas; aquí la generalización es más compleja y, por lo tanto, conlleva un coste cognitivo superior. Por ello, se deben prever intervenciones docentes que contribuyan a flexibilizar el razonamiento algebraico.

\section{Diseño de la experimentación}

Se proponen tareas con patrones geométricos que garanticen la actuación de los estudiantes, al menos en las sub tareas iniciales, mediante estrategias de resolución accesibles 
para ellos, que no impliquen desde el principio la simbolización. Las actividades exigen identificar una regla de formación: implícitamente, para determinar términos específicos en posiciones cercanas; explícitamente, para reconocer la regla que permita determinar términos alejados, para los cuales un procedimiento de recuento sería muy costoso y donde comunicar los resultados a través de la verbalización o simbolización es necesario.

Desde el punto de vista metodológico, el diseño es cuasi-experimental de grupo único con pre-test y post-test, de una hora de duración. Los test están constituidos por situaciones sobre recuento de patrones cuyo objetivo es reconocer los rasgos del razonamiento algebraico predominantes en los estudiantes y su evolución.

Dado que no hay asignación aleatoria de los sujetos a los grupos ni a los tratamientos (modos de enseñanza) y dado que las características del grupo, novedosas y muy específicas en la institución, no permiten la comparación ni con otro grupo transversal ni con una cohorte, el diseño es de validación interna; es decir, el análisis se centra en la confianza en la interpretación de la actividad matemática de los estudiantes, mediante un análisis cualitativo por estudio de casos.

En las Figuras 4 y 5 se muestran dos tareas de patrones y sus soluciones previstas, que sirven, aquí, de contraste (adaptaciones de GODINO et al., 2015a, 2015b). En la tarea del pretest se debe hallar la cantidad de cuadrados necesarios para dibujar un término particular de la sucesión; la relación corresponde a un modelo de función cuadrática (Figura 4).

En la solución 1 se realiza el recuento para los primeros términos, pero para los siguientes se establece la respuesta en función del lugar que ocupa el término en la serie, sin recurrir al recuento efectivo, utilizando un lenguaje simbólico. En la solución 2, el recuento se realiza descomponiendo la figura en dos, siendo una de ellas la figura anterior. Se representa el caso general simbólicamente y se realizan transformaciones con los símbolos. La solución 3 también es recursiva, pero el patrón identificado es otro: el número de cuadraditos se asocia con la suma de números impares. Aquí, la solución 3b, que aporta una demostración explícita de la suma de los $\mathrm{n}$ primeros números impares, se presume que formaría parte de una institucionalización a cargo del docente.

Así, las soluciones 1 y 3 a muestran rasgos de niveles de razonamiento 2, mientras que las soluciones 2 y $3 \mathrm{~b}$ muestran rasgos del nivel 3, al haber manipulado la escritura formal hasta una expresión más simple o la obtención formal de una igualdad, respectivamente. 
Considera la siguiente secuencia de cuadraditos.

\section{Tarea 1 (pre-test)}

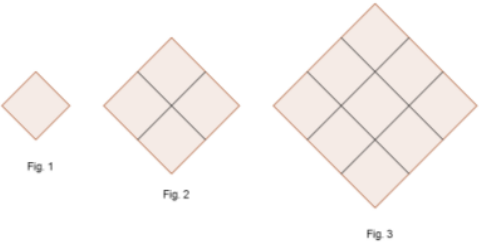

¿Cuántos cuadraditos se necesitan para formar la figura 5? ¿y para la figura 8? ¿y para la figura 30? ¿Cuántos cuadraditos se necesitan para formar la figura $n$ ?

\section{Soluciones previstas}

Solución 1: Sin necesidad de realizar un recuento, se establece una relación entre la cantidad de cuadraditos y el cuadrado del número de la figura.

Así, para la figura 1 se necesitan: $1^{2}$ cuadraditos; para la figura $2: 2^{2}$; para la figura $3: 3^{2}$; para la figura $4: 4^{2}$; para la figura 5:52; para la figura $8: 8^{2}$; para la figura $30: 30^{2}$; y para la figura $n: n^{2}$.

Solución 2: Otra forma de hacer el recuento es la siguiente:

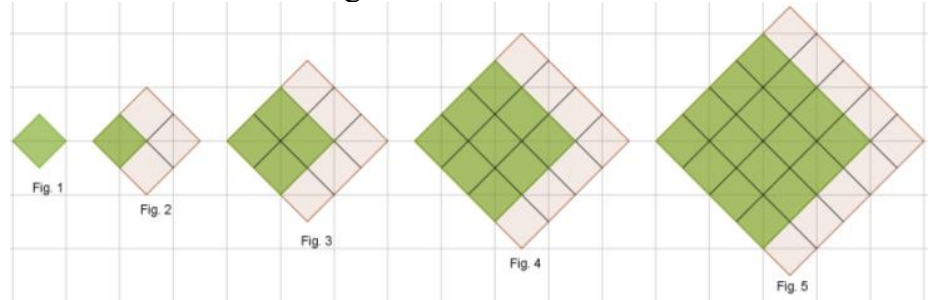

Se determina el número de cuadraditos que requiere cada término como la cantidad anterior más los que se necesitan para ampliar la figura; así, se obtiene que:

Para la figura 1 se necesita 1 ; para la figura $2: 1+3=4$; para la figura $3: 4+5=9$; para la figura $4: 9+7=16$; para la figura 5: $16+9=25$; para la figura $8: 7^{2}+(2(8)-1)=64$

Para la figura $\mathrm{n}:(n-1)^{2}+(2 n-1)=n^{2}$

Solución 3: Otra solución recursiva sería obtener, a partir de recuentos, las cantidades de cuadraditos para las primeras figuras:

$1 ; 1+3=4 ;(1+3)+5=9 ;(1+3+5)+7=16$; para la figura 5 serían $(1+3+5+7)+9=25 ; \ldots ;$

para la figura 8 serían: $(1+3+5+7+9+11+13)+15=64$. Así, a partir del recuento de estos casos, se tendría dos generalizaciones:

a) Basada en los resultados, por inducción empírica y sin demostración explícita: la suma de los $n$ primeros números impares es $n^{2}$.

b) Demostración aritmética-formalizada. Sea $S I_{n}$ la suma de los $n$ primeros números impares, entonces:

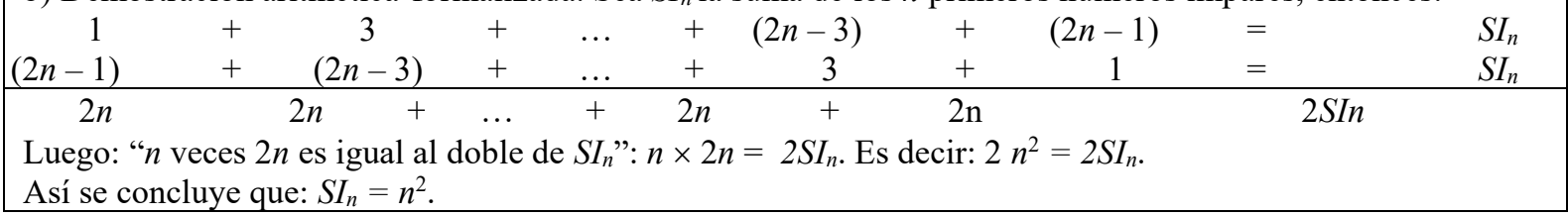

Figura 4 - Tarea de patrones en el pre-test y soluciones previstas Fuente: Elaboración propia de esta investigación

La tarea del post-test ${ }^{6}$ (figura 5) precisa determinar la cantidad de segmentos necesarios para formar la figura en una posición específica; responde a un modelo lineal. En todas las soluciones de la tarea 2 se plantea descomponer la figura; esto se puede hacer considerando los dos segmentos de los extremos y luego los demás, como ocurre en la solución 1, o considerando que está formada por una figura superior y otra inferior, ambas con la misma cantidad de segmentos, como en la solución 2; o bien, por último, considerando fijos tres segmentos en cada

\footnotetext{
${ }^{6}$ Para controlar la amenaza de sensibilización a la prueba, pre-test y post-test no son el mismo, sin embargo, estos test son equivalentes, puesto que se refieren al mismo contexto, contenidos matemáticos y tareas asociadas. Esta equivalencia posibilita el seguimiento de la actividad mostrada por los estudiantes.
} 
extremo y que en cada nuevo término el número total de segmentos se incrementa en 4.

Si bien en esta tarea no se solicita hallar el número de segmentos que permiten construir la figura $n$-ésima, se espera que la identificación de segmentos para la figura en la posición 20 se realice mediante la identificación de una regla general.

Considera la siguiente sucesión de figuras.

\section{Tarea 2 (post-test)}
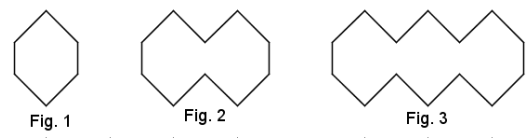

Si se mantiene el mismo patrón:

¿Cuántos segmentos se requieren para construir la figura 4? ¿y para la figura 5? ¿y para la figura 6?

¿Qué ocurre cada vez que construyes una nueva figura?, ¿notas alguna regularidad?

¿Cuántos segmentos se requieren para construir la figura 20 ?

\section{Soluciones previstas}

Solución 1: La figura 1 se forma con $2+4$ segmentos. En cada figura el número de segmento se incrementa en 4. La figura 4 se formará con $2+2(4)=10$ segmentos. La figura 20 se formará con $2+20(4)=82$ segmentos.

Solución 2: Otra forma de hacer el recuento para las figuras 4 y 5 es el siguiente:

Para la figura 6 , se necesitan $13 \times 2=26$

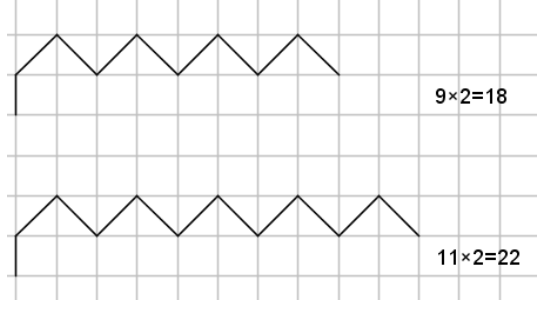

Para construir la fila superior de una figura se necesita el doble del número de la figura más uno, correspondiente a un segmento vertical y para la fila inferior se requiere una cantidad igual.

Para la figura 20 se necesitan $2(2(20)+1)$ para la fila superior y una cantidad similar para la inferior.

Para la figura $n$ se requieren $2(2 n+1)$ segmentos.

Solución 3: Otra forma de hacer el recuento es la siguiente: Considerar los 6 segmentos que forman la figura 1 e incrementar 4 segmentos más en cada nueva figura, ya que, para la figura 2 se necesitan $6+4$, es decir, 6+4(1); para la figura 3: 6+4(2); para la figura 6: 6+4(5). Así, para la figura 20 se necesitan $6+4(19)=82$.

Figura 5 - Tarea de patrones en el post-test y soluciones previstas Fuente: Elaboración propia de esta investigación

\section{Experimentación y resultados}

La investigación se realizó con seis estudiantes ${ }^{7}$ de 16-17 años, que habían culminado la Educación Secundaria en una escuela rural del Sistema Educativo Peruano. Durante la etapa escolar, el desarrollo formal de las Matemáticas, mediante expresiones simbólico-literales, fue limitado, con una prevalencia del registro oral, icónico o ideogramático, muy vinculado a usos y técnicas específicas. Los estudiantes iniciaban un curso propedéutico previo a sus estudios universitarios en carreras de humanidades. Así, la experiencia descrita se desarrolla en una

\footnotetext{
${ }^{7}$ Dado que no es objetivo de esta investigación analizar diferencias en las respuestas por razón de género, se utiliza el género masculino como género gramatical no marcado.
} 
asignatura de matemáticas que formó parte de un programa anual de inserción.

Tras el pre-test, se desarrolló un proceso de instrucción que constó de dos sesiones de trabajo, de dos horas cada una, en donde se abordaron actividades sobre identificación de patrones de formación de sucesiones de figuras geométricas. También se contemplaron tareas sobre identificación de patrones en secuencias numéricas y otras que requerían la manipulación de expresiones simbólicas y el reconocimiento de expresiones equivalentes.

De esta manera, se pretendía enfrentar a los estudiantes a actividades que exigieran identificar una regla de formación (unitarización), reconocerla implícitamente al tener que determinar términos específicos en posiciones cercanas (por ejemplo, 4 o 9) y también reconocer la regla, explícitamente, al tener que hallar términos más alejados (por ejemplo, 15 o 100), para los cuales un procedimiento de recuento sería muy costoso.

Esta actividad exigía comunicar los resultados a través de la verbalización o simbolización, aunque en ninguna de ellas se pidió expresamente la formulación simbólicoliteral del término n-ésimo. Las indicaciones de la actividad se dieron por escrito y los estudiantes trabajaron primero individualmente.

En ese proceso, los estudiantes elaboraron conjeturas y algunos de ellos las compartieron con el resto de la clase que las validaba o refutaba. Dos de los estudiantes identificaron formalmente el término n-ésimo y luego emplearon la expresión simbólica obtenida para determinar términos en posiciones lejanas. El docente tuvo a cargo el cierre de las discusiones. Este mismo esquema se repitió durante las dos sesiones. Finalmente, se aplicó el post-test.

A continuación, se presentan los resultados de cada estudiante a dos tareas, una del pretest y otra del post-test, se identifican los rasgos de razonamiento algebraico que muestran sus soluciones y se discuten teniendo en cuenta el proceso de instrucción desarrollado.

El estudiante A aborda la tarea 1 a través del recuento explícito de cuadraditos que formaban las primeras cuatro figuras (Figura 6); luego, sin recurrir, nuevamente, al recuento efectivo en la representación gráfica, determina el valor para las figuras 5 y 8 empleando lenguaje numérico. Si bien reconoce que el número de cuadraditos que se incrementan de figura en figura es igual al incremento anterior más 2, no consigue conjeturar una ley de formación por lo que no determina el número de cuadraditos necesarios para la figura 30. 


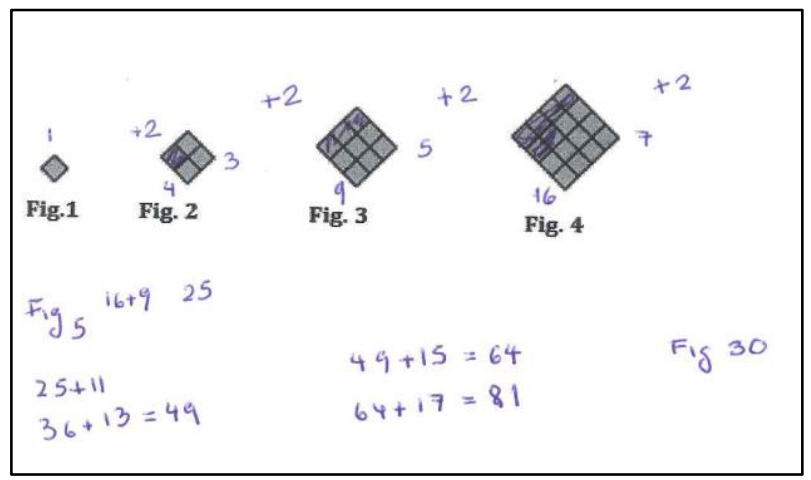

Figura 6 - Solución a la tarea 1 presentada por el estudiante A Fuente: Elaboración propia de esta investigación

El estudiante muestra un nivel 1 de algebrización, dado que, a partir de recuentos explícitos, basados en cálculos numéricos o figuras, es capaz de determinar valores de patrones específicos en función del lugar que ocupan en la serie, sin recurrir, nuevamente, al recuento efectivo (generalización cercana). Sin embargo, el lenguaje simbólico y formal está ausente.

El estudiante A, al resolver la tarea 2 del post-test indica que el número de segmentos necesarios para formar cada figura también se relaciona cuadráticamente con su posición, es decir, la respuesta en el post-test no se ajusta a la tarea, sino a la pregunta en el pre-test; no hay, pues, adaptación a la nueva situación. El proceso de estudio no ha permitido al estudiante A progresar en los distintos niveles de algebrización, quedándose en los dos primeros.

El estudiante $\mathrm{B}$, en la primera tarea cuenta el número de segmentos y no de cuadraditos (que es lo solicitado), mientras que en la segunda tarea donde sí se solicita el recuento de segmentos, cuenta las celdas de forma hexagonal. Es decir, adapta la respuesta de la tarea 2 según lo que se precisa en la 1 . Se observa, pues, un fenómeno de hipercorrección, ajustado a la norma del contrato pedagógico ${ }^{8}$ según la cual, la evaluación cumple una función de rendición de cuentas de los estudiantes al profesor sobre lo estudiado y aprendido.

En la Figura 7 se presenta la solución del estudiante $\mathrm{C}$ a la tarea 1, sin explicitar el recuento.

\footnotetext{
${ }^{8}$ En toda clase, hay reglas que determinan la relación profesor-estudiante. Aquellas generales, independientes del contenido, se dicen pedagógicas; aquellas que condicionan las acciones e intervenciones de los agentes en relación con el contenido, se dicen didácticas. El conjunto de reglas pedagógicas constituye el contrato pedagógico; el conjunto de reglas didácticas, el contrato didáctico. Estas nociones originales de la Teoría de Situaciones Didácticas en Matemáticas (BROUSSEAU, 1997) se han incorporado a diferentes enfoques teóricos, en particular al Ontosemiótico (Godino et al., 2009).
} 


$$
\begin{aligned}
& \text { para formar la figura } 5 \text { senecesitan } 25 \text { wadraditos } \\
& \text { La } 8 \text { senecesitan } 64 \\
& \text { La } 30 \text { se necesitan } 900 \\
& \text { Parala figura } n \text { se necesitan } n
\end{aligned}
$$

Figura 7 - Solución del estudiante $\mathrm{C}$ a la tarea 1

Fuente: Elaboración propia de esta investigación

El estudiante $\mathrm{C}$ identifica la relación entre el número de cuadraditos en función del lugar que ocupa la figura para casos particulares, mostrando rasgos de nivel 1 (generalización cercana $\left.^{9}\right)$ (Figura 8).

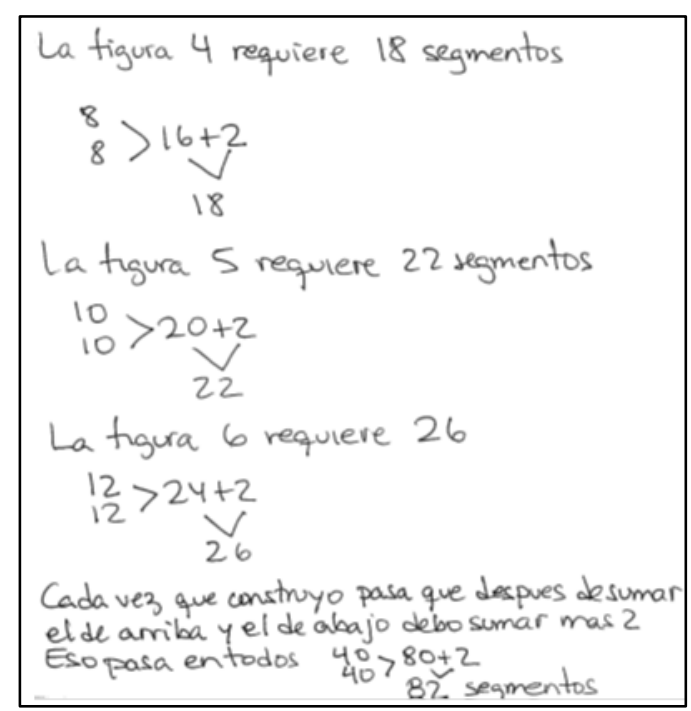

Figura 8 - Solución del estudiante $\mathrm{C}$ a la tarea 2 Fuente: Elaboración propia de esta investigación

Sin embargo, este estudiante no es capaz de expresar la solución general en lenguaje simbólico: para la figura $n$ [cualquiera] se necesitan $n$ [los que le correspondan]; de otra forma, el estudiante utiliza el símbolo $n$ como representante de un número: un lugar en la serie (la primera vez) o como representante de una cantidad (la segunda vez). Tras el proceso de instrucción, resuelve la pregunta 2 del post-test, empleando expresiones en lenguaje natural, que se refieren a objetos generales. Así, el estudiante establece relaciones entre el número de segmentos y el lugar que ocupa una figura en la serie, pero no de manera formalizada.

El estudiante D resuelve la tarea 1 empleando diferentes métodos (Figura 9): busca medios de control para verificar que su solución es correcta. Además, adapta sus procedimientos a la tarea, de modo que para determinar el número de cuadraditos que requiere la figura 30 opta solo por el método más eficiente; a saber: elevar al cuadrado. Finalmente, aporta una solución

\footnotetext{
${ }^{9}$ La generalización cercana constituye una norma del contrato didáctico en el contexto de las actividades con patrones, según la cual el análisis de un número finito de casos permite conjeturar reglas generales sobre las que habrá que argumentar posteriormente su validez (inducción empírica).
} 
general simbólico-literal. En resumen, el estudiante tiene un PMF de nivel 2 de algebrización, mostrando tres reglas fundamentales del contrato didáctico en las tareas con patrones; a saber: control sobre las respuestas, análisis de la eficacia de los métodos y búsqueda de la generalización mediante lenguaje simbólico literal.

\begin{tabular}{|c|c|c|}
\hline $1+3=4 \quad 2 \times 2=4$ & $3 \times 3=9$ & $4,4=16$ \\
\hline \multirow[t]{2}{*}{ 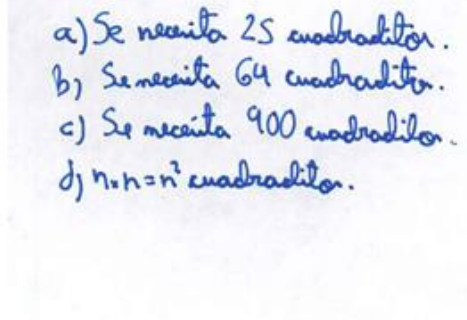 } & $\begin{array}{l}5 \\
25 \\
\frac{11}{36} \\
\frac{13}{494} \\
\frac{15}{64}\end{array}$ & $\begin{array}{l}30 x \\
\frac{30}{900}\end{array}$ \\
\hline & & $n \times n=n^{2}$ \\
\hline
\end{tabular}

Figura 9 - Solución del estudiante D a la tarea 1 Fuente: Elaboración propia de esta investigación

En la tarea 2, el estudiante D ya no utiliza varias formas. La consolidación del nivel algebraico y su operativización flexible permiten a dicho estudiante seleccionar los procesos según la tarea, desde un punto de vista de eficacia y coste. La ley de formación presentada muestra que ha considerado que cada hexágono aporta seis segmentos y luego se deben restar todos los segmentos que conectaron dos hexágonos, tal como se detalla en la Figura 10.

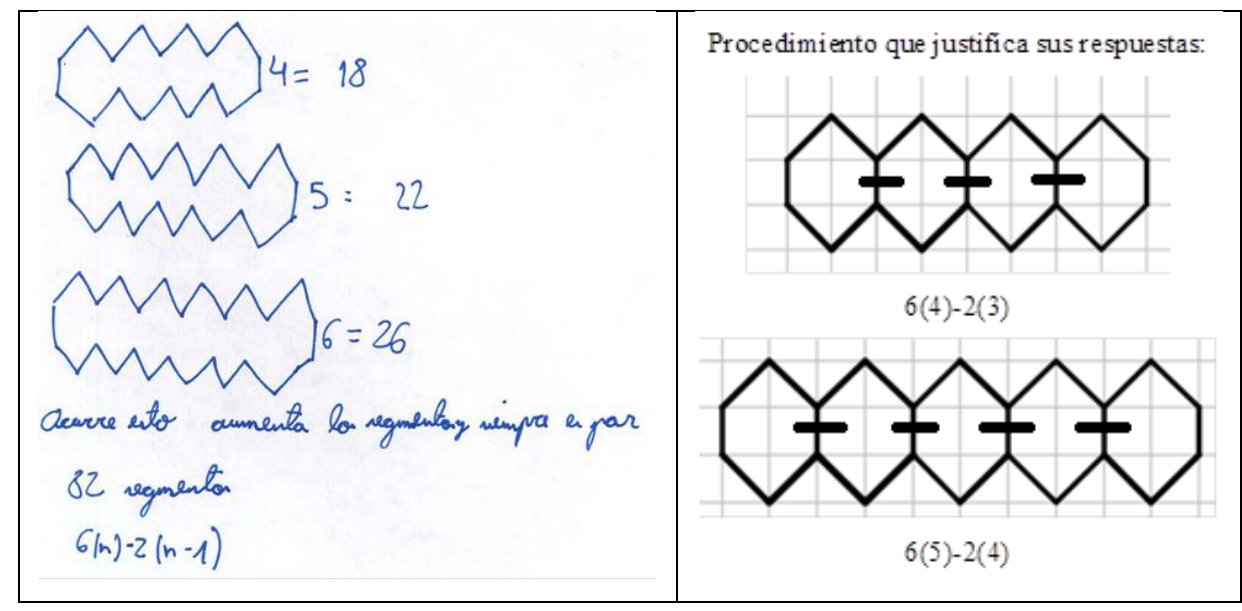

Figura 10 - Solución del estudiante D a la tarea 2

Fuente: Elaboración propia de esta investigación

El estudiante E reconoce la regla de formación mediante extensivos; presenta una solución que se basa en la solución 2, mostrada en la figura 5. En la generalización, intenta, sin éxito, una solución basada en el uso de expresiones que han formado parte del proceso instruccional. Así, se refuerza la tesis según la cual la cláusula del contrato pedagógico responder a las expectativas docentes es determinante para estos estudiantes, ya que, como en la respuesta del estudiante $\mathrm{B}$, se observa un fenómeno de hipercorrección; a saber, la solución 
que presenta el estudiante $\mathrm{E}$ a la tarea en el post-test es consistente con su respuesta en la del pre-test, sin muestra de evolución hacia la representación formal.

En resumen, la mayoría de los estudiantes ha podido identificar regularidades a partir de casos particulares y representaciones concretas. Estas regularidades les permiten determinar el valor de patrones específicos en función del lugar que ocupan en la serie. Sin embargo, excepto uno de ellos, no muestran aún recursos para determinar la regla general de formación de manera explícita mediante lenguaje simbólico ni menos aún la realización de su demostración.

\section{Propuesta de intervención para el desarrollo del RAE mediante patrones}

La ingeniería didáctica (ARTIGUE, 1989) y, en general, las investigaciones basadas en el diseño (GODINO et al., 2014b), permiten, a partir del contraste entre los análisis a priori y a posteriori, extraer conclusiones para el diseño y puesta en marcha de prácticas matemáticas mejor adaptadas al sistema didáctico.

En este trabajo, el pre-test y el post-test aportan información sobre las dimensiones cognitiva e instruccional; en concreto, aportan datos explícitos sobre el progreso de los aprendizajes y sobre el impacto en estos del proceso instruccional puesto en marcha. Así, el post-test aporta información del término del proceso instruccional y permite el contraste entre lo previsto (según las restricciones cognitiva e institucional) y lo observado o contingente.

De esa manera, se obtienen criterios para la intervención, que han sido fundamentados teóricamente y contrastados experimentalmente. De forma sucinta, se puede aceptar el estudio previo (secciones 1-4) como el análisis a priori que permite la determinación de la propuesta que se describe a continuación, cuya aplicación y contraste aportará a su consistencia interna, así como aspectos de mejora para el control del funcionamiento del sistema didáctico.

Para ejemplificar esta propuesta se retoma la tarea de recuento inicial, añadiendo una tercera pregunta: determina cuántos segmentos se requerirán para formar la figura 687. Los estudiantes podrán responder al primer apartado (segmentos en la figura 5) con una actividad puramente aritmética, realizando recuentos. Para responder al segundo y tercer apartado (segmentos en las figuras 87 y 687, respectivamente) tendrán que optar por la búsqueda de un patrón específico en función del lugar que ocupa el término en la serie o por soluciones aritméticas que involucren recuentos eficientes.

Para el primer apartado, podría darse alguna de las soluciones presentadas en la figura 5. Por ejemplo, se podría reconocer que la figura 87 se forma con $4(87)+2$ segmentos y la figura 
687 con 4(687)+2 segmentos. En este caso, el siguiente paso en la intervención docente sería promover el desarrollo del lenguaje simbólico, que diera paso a los niveles 2 y 3 de algebrización. Este progreso puede basarse en la conexión de agrupamientos, puesto que es una estrategia de base (BROUSSEAU, 1997) que conocen los estudiantes. Se realizaría en los siguientes pasos.

Paso 1. Construcción de una tabla con el número de segmentos necesarios en todos los casos comprendidos entre 1 y 10, así como en los primeros múltiplos de 10 (Tabla 1).

Tabla 1 - Segmentos en figuras de base.

\begin{tabular}{lllllllllllll}
\hline Figura & 1 & 2 & 3 & 4 & 5 & 6 & 7 & 8 & 9 & 10 & 100 & 1000 \\
\hline Segmentos & 6 & \multirow{2}{*}{10} & \multirow{2}{*}{14} & \multirow{2}{*}{18} & 22 & 26 & 30 & \multirow{2}{*}{34} & \multirow{2}{*}{38} & 42 & 402 & 4002 \\
\hline
\end{tabular}

Fuente: Elaboración propia de esta investigación

Paso 2. Descomposición polinómica del número. Por ejemplo: $687=6 \times 100+8 \times 10+7$.

Paso 3. Cálculo de los segmentos que aportan las agrupaciones necesarias y supresión de los segmentos de los empalmes; a saber: 2 por el número de empalmes, siendo este número la suma de agrupaciones de potencias de 10 necesarias, excluyendo las unidades ${ }^{10}$. Así, para el cálculo de los segmentos necesarios para formar la figura que ocupa el lugar 687:

$$
\underbrace{\mathbf{6} \times 402}_{\begin{array}{c}
\text { segmentos } \\
\text { centenas }
\end{array}}+\underbrace{\mathbf{8} \times 42}_{\begin{array}{c}
\text { segmentos } \\
\text { decenas }
\end{array}}+\underbrace{\mathbf{3 0}}_{\begin{array}{c}
\text { segmentos } \\
\text { unidades }
\end{array}}-\underbrace{2 \times(\mathbf{6}+\mathbf{8})}_{\text {empalmes }}=2750
$$

Además, la construcción de la Tabla 1 permite conjeturar que para la figura siguiente se necesitan 4 segmentos más que en la anterior $\mathrm{y}$, al menos para las potencias de 10, que se necesitan cuatro por la potencia $(10,100$ etc.) más 2. La sistematización en la construcción de la tabla permite la determinación de propiedades generales y la propuesta de una escritura (descomposición polinómica de números) que sirve de base a otras más formales.

Paso 4. A partir del método de recuento por conexión de agrupamiento, se introduce la inducción matemática: ¿cómo se relacionan dos figuras consecutivas en la serie? (Figura 12).

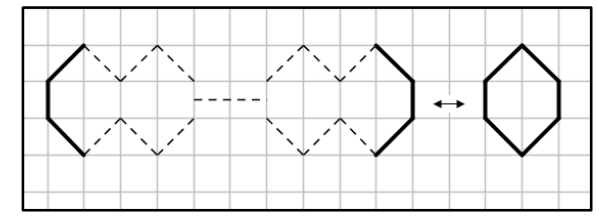

Figura 12 - Recuento por conexión de agrupamientos entre dos figuras consecutivas Fuente: Elaboración propia de esta investigación

Los estudiantes establecerán que la relación es sumar 4 segmentos a los necesarios en

\footnotetext{
${ }^{10}$ En lo que sigue se utilizará por simplicidad potencias de 10 como sinónimo de potencias de 10, excluyendo la unidad, es decir: $10^{n}, n>0, n \in \mathbf{N}$.
} 
la figura anterior, relación que el docente formalizará mediante una regla de recurrencia:

$$
\left\{\begin{array}{l}
a_{n+1}=a_{n}+4, \forall n \in \mathbb{N}, n \geq 1 \\
a_{1}=6
\end{array}\right.
$$

Los estudiantes verifican entonces su validez para valores particulares: $a_{1}=6 ; a_{2}=a_{1}+4=$ $6+4=10 ; a_{3}=a_{2}+4=6+4+4=14 ; a_{4}=a_{3}+4=6+4+4+4=18$ etc. Estos cálculos motivan la determinación de una fórmula general que no sea dada de forma recurrente, ya que la aplicación de ésta supone, para términos suficientemente alejados del primero, una cadena de cálculos larga y tediosa, que además puede comportar errores. Así, el docente solicitará una escritura con producto, esto es, en lugar de escribir el resultado de la forma: $a_{1}=$ 6 у $a_{2}=a_{1}+4=6+4$, se dejan las operaciones indicadas, de modo que $a_{3}$ se puede escribir así:

$$
a_{3}=a_{2}+4=6+4+4=6+\underline{2 \times 4} \text { [El docente subraya } 2 \times 4 \text { en la pizarra] }
$$

Se plantea como nueva tarea escribir $a_{4}, a_{5}$ y $a_{6}$ con producto y analizar si hay alguna propiedad que se cumple siempre. ¿Cómo se calculará $a_{87}$ ?’. Los estudiantes concluirán que: $a_{87}=6+4 \times 86$.

Así, consolidado el nivel 1 de algebrización, el docente devuelve la tarea a los estudiantes, solicitando una expresión para un término cualquiera ( $n$-ésimo). La actividad se desarrolla mediante una situación dialógica que permita la evolución de la escritura hasta una simbólica $a_{n}=6+4(n-1)$. Antes de esta escritura formal, los estudiantes harán formulaciones en lenguaje natural del tipo: hay que multiplicar el anterior por cuatro y sumarle seis, donde, por un lado, se omitirá la estructura funcional (el término n-ésimo es igual...) y, por otro lado, se confundirá un término y el lugar que ocupa (de ahí que se diga el anterior).

Una vez determinada la expresión $a_{n}=6+4(n-1)$, se realizan manipulaciones, hasta obtener la expresión: $a_{n}=4 n+2$. Este proceso supone el progreso hasta el nivel 3 de algebrización, puesto que requiere de la manipulación simbólica para la obtención de una expresión equivalente sin referencia explícita al patrón. Aquí, es necesario observar que, recíprocamente, una fórmula se puede representar mediante construcciones distintas.

\footnotetext{
While it is true that a given pictorial pattern can be interpreted in a variety of ways through different modes of visual deconstruction, each leading to its own algebraic expression of generality, it is equally true, though often left unexplored, that a single algebraic expression could conversely have arisen from different modes of visualization (SAMSON, 2011, p. 32).
}

Paso 5. Se propone demostrar la fórmula hallada por el principio de inducción matemática. De esta manera, se progresará en el uso del lenguaje simbólico y de expresiones equivalentes. Esta manipulación progresa hasta el nivel 5 de algebrización, ya que intervienen parámetros y 
variables (Godino et al., 2015a).

De hecho, en el caso de la inducción, la letra $n$ significa un parámetro (cuando se demuestra la regla general y se manipula el valor de $n$ como un caso concreto, pero genérico; para cada $n$ ) y a la vez una variable (cuando se ha demostrado la corrección de la regla general y se utiliza como función). Evidentemente, este salto del nivel 3 al 5 es brusco y requiere de una especial vigilancia del docente, el cual puede apoyarse en el recuento por conexión de dos figuras consecutivas (Figura 12).

Paso 6. Por último, se compara el coste y eficacia de la utilización de expresiones recursivas $a_{n+1}=a_{n}+4\left(a_{1}=6\right)$ y de la regla general $a_{n}=4 n+2$, enfatizando las limitaciones de la primera. Esto se puede hacer evidente si se solicita a los estudiantes que empleen ambas para hallar el número de segmentos necesarios para construir la figura 687.

Finalmente, la eficacia en la resolución de tareas, la consolidación en el uso gradual de los lenguajes, la adquisición de los distintos objetos y de su distinto carácter (particular/general), así como el progreso de la práctica según los procesos involucrados (particularización, generalización, unitarización y reificación), requieren la rutinización en la resolución de las tareas. Así, es necesario proponer situaciones diversas sobre patrones que involucren los procedimientos introducidos y permitan afianzar los aprendizajes adquiridos.

\section{Síntesis, conclusiones y cuestiones abiertas}

Las situaciones de recuento con patrones son un contexto apropiado para el desarrollo del Razonamiento Algebraico Elemental (RAE). Dependiendo del término solicitado en una serie, los recursos necesarios varían desde la representación efectiva y el simple conteo a la necesidad de determinación de reglas generales; desde el lenguaje natural, icónico y meramente aritmético al lenguaje simbólico-literal y su manipulación sintáctica; desde la prueba empírica con extensivos a la demostración formal. Por ello, es posible proponer situaciones que permitan diagnosticar el nivel algebraico de estudiantes y determinar procesos de estudio potenciales para su desarrollo y afianzamiento.

Aquí es preciso observar que la maestría de un sujeto no se valora, únicamente, por la eficacia, sino también por la adecuación de la resolución a la tarea propuesta. El Pensamiento Matemático Flexible (PMF) es la capacidad del sujeto de adecuar su resolución a las trasformaciones de objetos y a lenguajes necesarios para la resolución de la tarea.

El análisis a priori de tareas de recuento determina indicadores de reconocimiento de prácticas matemáticas estandarizadas, que sirven para el análisis del desempeño en la resolución 
de estudiantes. En particular, en los estudiantes de la muestra se observa un desarrollo de los niveles 0 (aritmético) y 1 (de iniciación algebraica) hacia la consolidación estable y fiable de este último nivel y la identificación de rasgos propios del nivel 2, aunque, en general, sin un uso adecuado del lenguaje simbólico-literal. Es aquí donde la intervención del docente debe poner el énfasis.

El progreso en la manipulación de intensivos (objetos generales) con lenguaje formal no puede ser el punto de partida, sino la síntesis de un trabajo que parta de la actividad concreta propia de los primeros niveles $(0,1$ y 2$)$. Por ello, la propuesta de intervención articula en seis etapas el paso de los recuentos efectivos de los primeros elementos de las series de patrones a la demostración formal mediante inducción matemática y el análisis de escrituras equivalentes en la construcción del patrón. Además, dada la complejidad del proceso de estudio, se supone la reiteración del procedimiento en distintas situaciones, hasta la rutinización en contextos diversos donde la manipulación de intensivos es explícitamente necesaria.

El análisis de casos permite, además, identificar ciertos comportamientos ajustados al funcionamiento del sistema didáctico ya sea el contrato didáctico (respuestas condicionadas por actividad matemática previa) o el contrato pedagógico (respuestas basadas en saberes aprendidos como forma de mostrar al profesor que el estudiante ha estudiado). Estos resultados resaltan la importancia de las normas (GODINO et al., 2009) en los procesos de estudio.

Esto es especialmente importante en la experimentación, ya que, por un lado, las prácticas matemáticas previas de los sujetos se han centrado en usos contextualizados, concretos y prácticos y, por otro lado, el cambio de institución de referencia les exige una adecuación rápida, tanto a los contenidos como a los requerimientos del profesorado, condicionados estos por la institución.

Reconocimiento: Trabajo realizado en el marco del proyecto Elaboración de una propuesta de desarrollo del razonamiento algebraico elemental para docentes (Referencia CAP472/PUCP).

\section{Referencias}

ARTIGUE, M. Ingénierie didactique. Recherches en Didactique des Mathématiques, Grenoble, v. 9 , n. 3, p. 281-308, 1989.

BLANTON, M. L.; KAPUT, J. J. Functional Thinking as a Route Into Algebra in the Elementary Grades. In: CAI, J; KNUTH, E (Ed.). Early Algebraization, Advances in Mathematics Education. Heidelberg: Springer-Verlagp, 2011. p. 5-23.

BROUSSEAU, G. The Theory of didactical situation in mathematics. 1st ed. Dordrech: Kluwer, 1997.

CALLEJO, M.; ZAPATERA, A. Flexibilidad en la resolución de problemas de identificación de 
patrones lineales en estudiantes de secundaria. BOLEMA, Rio Claro, v. 28, n. 48, p. 64-88, 2014.

GASCÓN, J.; BOSCH, M.; RUIZ-MUNZÓN, N. El problema del álgebra elemental en la teoría antropológica de lo didáctico. En: MUÑOZ-ESCOLANO, J. M. et al. (Ed.). Investigación en Educación Matemática XXI. Zaragoza: SEIEM, 2017. p. 25-47.

GODINO J. et al. Aproximación a la dimensión normativa en didáctica de las matemáticas desde un enfoque ontosemiótico. Enseñanza de las Ciencias, Barcelona, v. 27, n. 1, p. 59-76, 2009.

GODINO J. D. et al. Naturaleza del razonamiento algebraico elemental. BOLEMA, Rio Claro, v. 26, n. 42B, p. 483-511, 2012.

GODINO, J. D, et al. Niveles de algebrización de la actividad matemática escolar. Implicaciones para maestros. Enseñanza de las Ciencias, Barcelona, v. 32, n. 1, p. 199-219, 2014 a.

GODINO, J. D. et al. Ingeniería didáctica basada en el enfoque ontológico - semiótico del conocimiento y la instrucción matemáticos. Recherches en Didactique des Mathématiques, Grenoble, v. 34, n. 2/3, p. 167-200, 2014 b.

GODINO, J. D. et al. Niveles de algebrización de las prácticas matemáticas escolares. Articulación de las perspectivas ontosemiótica y antropológica. Avances de Investigación en Educación Matemática, Madrid, n. 8, p. 117-142, 2015a.

GODINO, J. D. et al. Evaluación de conocimientos didáctico - matemáticos sobre razonamiento algebraico elemental de futuros maestros. Revista de Educación, Madrid, n. 370 , p. $199-228,2015$ b.

GODINO, J. D.; BURGOS, M. Perspectiva ontosemiótica del razonamiento algebraico escolar. En: MUÑOZ-ESCOLANO, J. M. et al. (Ed.). Investigación en Educación Matemática XXI. Zaragoza: SEIEM, 2017. p. 49-66.

KAPUT, J.; CARRAHER, D. W.; BLANTON, M. L. Algebra in the early grades. 1st ed. New York: Routledge, 2008.

KIERAN, C. et al. Early Algebra. 1st ed. Hamburg: Springer, 2016.

MOSS, J.; LONDON, S. An Approach to Geometric and Numeric Patterning that Fosters Second Grade Students' Reasoning and Generalizing about Functions and Co-variation. In: CAI, J.; KNUTH, E. (Ed.). Early Algebraization, Advances in Mathematics Education. Heidelberg: Springer-Verlag, 2011. p. 277-302.

SAMSON, D. Capitalising on inherent ambiguities in symbolic expressions of generality. The Australian Mathematics Teacher, Adelaide, v. 67, n. 1, p. 28-32, 2011.

WILHELMI, M. R.; GODINO, J. D.; LACASTA, E. Configuraciones epistémicas asociadas a la noción de igualdad de números reales. Recherches en Didactique des Mathématiques, Grenoble, v. 27, n. 1, p. 77-120, 2007a.

WILHELMI, M. R.; GODINO, J. D.; LACASTA, E. Didactic effectiveness of mathematical definitions. The case of the absolute value. International Electronic Journal of Mathematics Education, London, v. 2, n. 2, p.72-90, 2007b.

Submetido em 23 de Fevereiro de 2018. Aprovado em 22 de Agosto de 2018. 